\title{
Hiperplasias epiteliais em espécimes de mamoplastia redutora estética bilateral e mamoplastia redutora contralateral a câncer de mama
}

\author{
Epithelial hyperplasia in specimens from bilateral reduction aesthetic mammaplasty and reduction \\ mammaplasty contralateral to breast cancer
}

Luciene Simões de Assis Tafuri'; Helenice Gobbi ${ }^{2}$

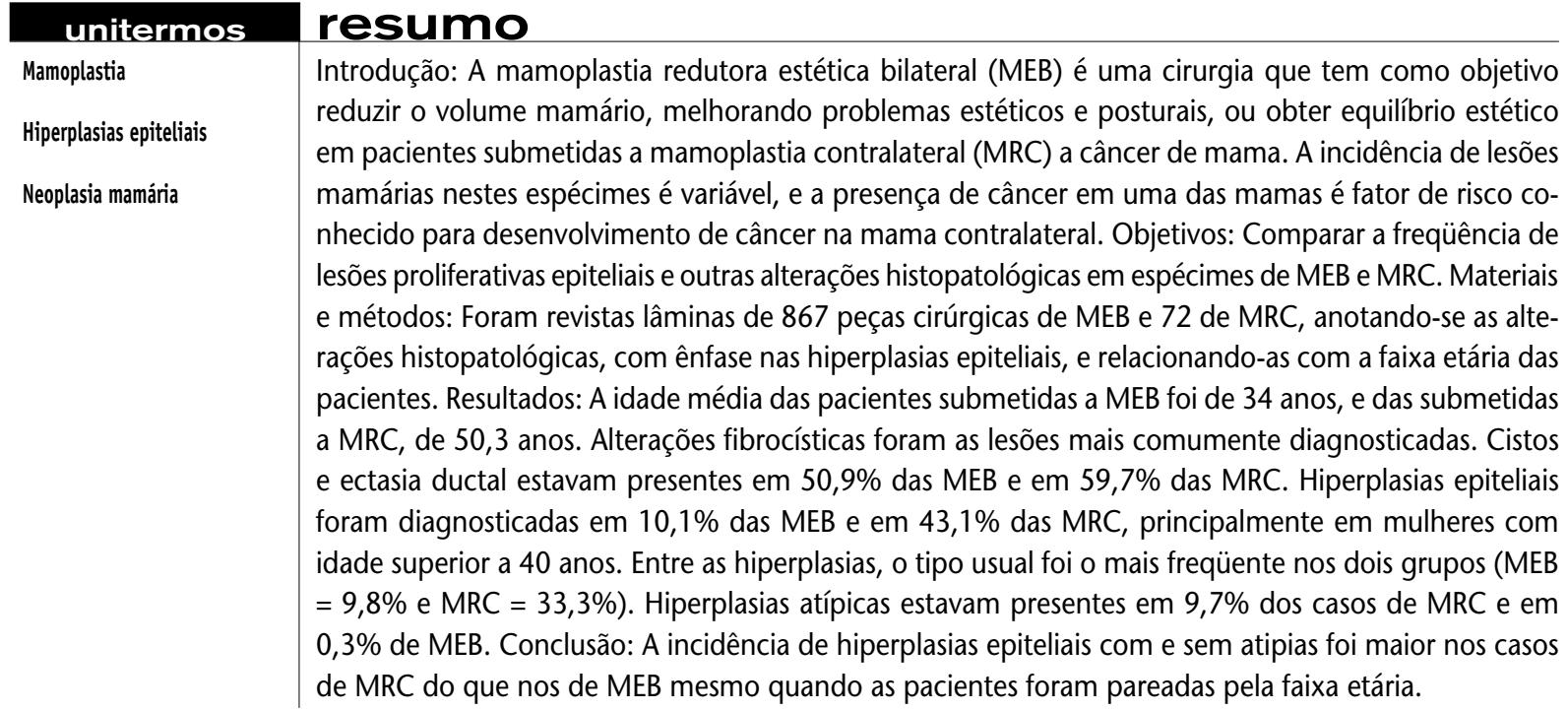

abstract

Background: Reduction mammaplasty is a surgery whose objective is to reduce the mammary size, improving aesthetic (MEB), or to attain of aesthetic balance in patients submitted to mammaplasty contralateral to breast cancer (MRC). The incidence of breast lesions in these specimens is variable and the previous cancer in one breast is a known risk factor for cancer in the contralateral breast. Aims: To compare the frequency of epithelial proliferative breast lesions and other histological changes in specimens of MEB and MRC. Methods: Breast surgical specimens from 867 MEB and 72 MRC had been reviewed and histological changes evaluated, with emphasis in epithelia hyperplasias relating them to patient's age. Results: The average age of patients was $M E B=34$ years and MRC $=50.3$ years. Fibrocystic change was the most common diagnosis. Cysts/duct estasia were present in $50.9 \%$ of MEB and $59.7 \%$ of MRC. Epithelial hyperplasia was diagnosed in $10.1 \%$ of MEB and $43.1 \%$ of MRC specimens, especially in women over 40 years. Usual hyperplasia was the most frequent type in both groups (MEB $=9.8 \%$ and $M R C=33.3 \%)$. Atypical hyperplasia was present in 0.3\% MEB and $9.7 \%$ MRC cases. Conclusions: The incidence of epithelial hyperplasias was higher in MRC than in MEB specimens in spite of age. key words

Mammaplasty

Hyperplasia

Breast neoplasm

1. Doutoranda do Programa de Pós-Graduação em Patologia da Faculdade de Medicina da Universidade Federal de Minas Cerais (UFMG).

2. Professora-adjunta-doutora do Departamento de Anatomia Patológica e Medicina Legal da Faculdade de Medicina da UFMG.

Trabalho realizado no Laboratório de Patologia Mamária do Departamento de Anatomia Patológica e Medicina Legal da Faculdade de Medicina da UFMG, Belo Horizonte (MG).

Apoio: Fundação de Amparo à Pesquisa do Estado de Minas Cerais (FAPEMIC) (CDS 560/01) e Conselho Nacional de Desenvolvimento Científico e Tecnológico (CNPq) (520117/00-0 NV).

Este trabalho é parte da tese de doutorado intitulada Marcadores Moleculares em Hiperplasias Epiteliais Mamárias de Pacientes Submetidas à Mamoplastia Redutora Estética Bilateral e Contralateral a

Câncer de Mama. Resultados parciais deste trabalho foram apresentados no XXIV Congresso Latino-Americano de Patologia (apresentação oral), em Quito, Equador, de 20 a 24 de outubro de 2003. 


\section{Introdução}

A hipertrofia mamária é caracterizada pela progressão volumétrica, em geral bilateral, das mamas, devido principalmente ao aumento predominante do estroma e, em parte, do parênquima glandular. A etiologia da hipertrofia mamária não é bem esclarecida, mas pode estar relacionada a fatores genéticos e hormonais, resultando em excessiva sensibilidade hormonal e conseqüente hipertrofia do componente estromal e do epitélio glandular da mama ${ }^{(4)}$.

No Brasil, as portadoras de hipertrofias mamárias volumosas buscam com freqüência tratamento cirúrgico para redução das mamas. A mamoplastia estética redutora bilateral (MEB) é uma das cirurgias da mama mais comuns em nosso meio ${ }^{(12)}$. A cirurgia tem como objetivo reduzir o volume mamário, melhorando a estética e auxiliando na correção de problemas posturais, dorsalgia e ptose, principalmente após gravidez e lactação( ${ }^{(1)}$. A mamoplastia tem sido utilizada também para a obtenção de equilíbrio estético ou postural em pacientes submetidas a mastectomia ou setorectomia/ quadrantectomia contralateral por câncer de mama( ${ }^{(7)}$.

A maioria das pacientes que buscam o atendimento médico para a realização da mamoplastia redutora estética não apresenta qualquer alteração ao exame físico ou radiológico das mamas. As análises macro e microscópica das peças cirúrgicas podem permitir o diagnóstico de alterações fibrocísticas, bem como de lesões proliferativas pré-malignas ou a identificação de câncer oculto de mama não-detectado no pré-operatório.

A incidência de lesões mamárias em espécimes de mamoplastia redutora estética ou contralateral é variável ${ }^{[12,28,29,}$ 34). Cerca de $60 \%$ das mulheres categorizadas como normais clinicamente apresentam alguma alteração histológica nos espécimes de mamoplastia em pelo menos uma de suas mamas $^{(1)}$. A maioria destas alterações é composta por lesões benignas não-neoplásicas, como as alterações fibrocísticas incluindo cistos, metaplasia apócrina, adenose e eventuais fibroadenomas ${ }^{(12,28)}$. Por outro lado, o câncer oculto é raro em pacientes assintomáticas submetidas a MEB, com incidência variando entre $0,06 \%$ e $4 \%(1,33,34)$.

A presença de câncer em uma das mamas é um fator de risco conhecido para o desenvolvimento de câncer na mama contralateral $(2,19)$. A incidência de câncer contralateral varia de $1,2 \%$ a $14 \%$, dependendo do tipo de população e do seguimento avaliado ${ }^{(10)}$. Ele pode ser sincrônico, com diagnóstico simultâneo, ou metacrônico, quando ocorre algum tempo depois ${ }^{(6)}$. Em algumas pacientes, a deteç̧ão do tumor contralateral ocorre quando se realiza a mamoplastia redutora contralateral (MRC), que é feita para se obter simetria após mastectomia e/ou quadrantectomia, ou ainda nas pacientes submetidas a mastectomia contralateral profilática ${ }^{(22,27)}$.

A análise criteriosa dos espécimes de MEB e MRC pode permitir a detecção de lesões proliferativas pré-malignas ou de câncer oculto em pacientes submetidas a cirurgia estética ou naquelas com risco clínico aumentado de desenvolver um segundo carcinoma na mama contralateral.
Neste trabalho comparamos a freqüência de lesões proliferativas epiteliais e outras alterações histopatológicas em espécimes de mamoplastia estética bilateral e em espécimes de mamoplastia redutora contralateral a câncer de mama.

\section{Metodologia}

Foram revistas 939 peças cirúrgicas de mamoplastia provenientes de cirurgias realizadas no Hospital das Clínicas da Universidade Federal de Minas Gerais (HC/UFMG) em Belo Horizonte, Minas Gerais, no período de janeiro de 1994 a maio de 2003. Dados clínicos das pacientes foram obtidos a partir das requisições de exame e dos prontuários médicos. Os dados referentes aos espécimes foram coletados dos laudos anatomopatológicos arquivados no Laboratório de Patologia Mamária do HC/UFMG. Nos casos de mamoplastias estéticas bilaterais, são amostrados, de rotina, quatro fragmentos de cada espécime e, se necessário, amostram-se áreas adicionais. Nos casos de MRC amostram-se, no mínimo, oito fragmentos e, se preciso, são incluídas outras áreas.

As lâminas foram revisadas por dois examinadores, preenchendo-se um protocolo específico ${ }^{(14)}$ discriminando as alterações histopatológicas encontradas. As lesões benignas não-neoplásicas, como cistos, metaplasia apócrina, adenose e adenose esclerosante, quando presentes, foram computadas individualmente. Para a classificação das hiperplasias epiteliais foram empregados os critérios propostos por Page e Anderson (1987) ${ }^{(25)}$ e a nova nomenclatura da Organização Mundial da Saúde (OMS) (2003) ${ }^{(36)}$. A nova classificação da OMS divide as lesões proliferativas intraductais em hiperplasia ductal usual (HDU), hiperplasia ductal atípica (HDA) e carcinoma ductal in situ (CDIS). Nesta classificação, as hiperplasias ductal leve e ductal moderada/florida de Page e Anderson são agrupadas em HDU sem atipias. Diferente da terminologia adotada por Page e Anderson, na classificação da OMS de 2003 a hiperplasia lobular atípica é classificada juntamente com o carcinoma lobular in situ como neoplasia lobular.

Foram analisados a idade das pacientes, o peso dos espécimes cirúrgicos e a freqüência das alterações histopatológicas, com ênfase nas hiperplasias epiteliais. Os dados foram armazenados e analisados empregando-se o programa Epi Info 6.0.

\section{Resultados}

Dos 939 casos, 867 eram mamoplastias estéticas bilaterais e 72 mamoplastias redutoras unilaterais em pacientes submetidas a mastectomia e setorectomia/quadrantectomia por carcinoma na mama contralateral. Os pesos das peças cirúrgicas variaram de 16 a 2.600 gramas (média = MRC: $361,7 \mathrm{~g} \pm 352,2 \mathrm{~g}$ e MEB: $458,4 \mathrm{~g} \pm 331 \mathrm{~g}$ ). A idade das pacientes submetidas a MEB variou de 15 a 78 anos (média = $34 \pm 12,8$ anos), e das submetidas a MRC, de 23 
a 73 anos (média $=50,3 \pm 10,5$ anos). A média de idade foi significativamente superior nas pacientes submetidas a MRC (Tabela 1).

As lesões benignas foram mais freqüentes na mama esquerda do que na direita, tanto nas pacientes submetidas a MEB (mama esquerda $=50,6 \%$ e mama direita $=49,4 \%$ ) como naquelas submetidas a MRC (mama esquerda = $58,3 \%$ e mama direita $=42,9 \%$ ). A maioria das pacientes (80\%) do grupo de MEB foi submetida a mamoplastia devido à hipertrofia mamária, sendo que 7,4\% delas apresentavam, além de hipertrofia, ptose das mamas.

Alterações fibrocísticas estavam presentes nos espécimes dos dois grupos, sendo que a maior parte das pacientes apresentou mais de um tipo de alteração histopatológica no mesmo espécime de mamoplastia. Os cistos/ectasia ductal foram as alterações mais freqüentes (MRC $=59,7 \%$ e MEB $=50,9 \%$ ). A distribuição dos diagnósticos histopatológicos nos dois grupos está sumarizada na Tabela 2.

As hiperplasias epiteliais foram diagnosticadas em 31 de 72 casos $(43,1 \%)$ de MRC e em 88 de 867 casos $(10,1 \%)$ de $M E B$, principalmente em mulheres com idade superior a 40 anos nos dois grupos.

Entre as hiperplasias, o tipo usual (moderada/florida) foi o mais freqüente nos dois grupos (MRC $=77,5 \%$ e $\mathrm{MEB}=$ $96,3 \%)$. Nos espécimes de MRC foram encontrados quatro casos de HDA e cinco de hiperplasia lobular atípica/neoplasia lobular (HLA/NL), sendo que dos casos contendo hiperplasias atípicas, dois apresentavam tanto HDA como $\mathrm{NL} / \mathrm{HLA}$ (Figura). Nos espécimes de MEB foram observados três casos de NL/HLA e nenhum de HDA (Tabela 3). Nos cinco casos classificados como NL/HLA, a lesão lobular era pouco extensa, não expandindo completamente mais de $50 \%$ das unidades lobulares, preenchendo os critérios descritos por Page et al. como HLA, e não como carcinoma lobular in situ.

Os tipos de câncer encontrados na mama contralateral à MRC foram os carcinomas ductais invasor e in situ em 34 casos (42,7\%), o carcinoma lobular invasor e o carcinoma
Distribuição segundo a faixa etária das mulheres submetidas a MRC e

Tabela 1 a MEB

\begin{tabular}{lcc} 
Faixa etária & MRC & MEB \\
$<20$ anos & $0(0 \%)$ & $94(10,8 \%)$ \\
$20-29$ anos & $2(2,8 \%)$ & $236(27,2 \%)$ \\
$30-39$ anos & $7(9,7 \%)$ & $194(22,4 \%)$ \\
$40-49$ anos & $21(29,2 \%)$ & $140(16,1 \%)$ \\
$50-59$ anos & $24(33,3 \%)$ & $73(8,4 \%)$ \\
$>60$ anos & $12(16,7 \%)$ & $30(3,5 \%)$ \\
Sem informação & $6(8,3 \%)$ & $100(11,5 \%)$ \\
Total & $\mathbf{7 2 ( 1 0 0 \% )}$ & $\mathbf{8 6 7 ( 1 0 0 \% )}$ \\
\hline
\end{tabular}

lobular in situ em dez casos (13,8\%), um caso de carcinoma medular $(1,3 \%)$ e dois carcinomas colóides $(2,6 \%)$. Em 25 casos $(34,7 \%)$ não foi possível obter a informação do subtipo de câncer diagnosticado, porque o espécime foi examinado em outro laboratório e a paciente, encaminhada ao nosso hospital apenas para a cirurgia de mamoplastia.

\section{Discussão}

Neste trabalho avaliamos a freqüência de hiperplasias epiteliais e outras alterações histopatológicas em peças cirúrgicas de MEB e MRC. O estudo dos espécimes obtidos de mamoplastia estética nos permitiu diagnosticar lesões benignas não-neoplásicas e lesões proliferativas consideradas pré-malignas da mama em espécimes de mamoplastia de pacientes assintomáticas. Nossos achados são semelhantes aos descritos por outros autores ${ }^{(12,28,29,32,34)}$.

Em nosso estudo demos ênfase à detecção das hiperplasias epiteliais que estão associadas a risco aumentado para

Tabela 2 Incidência de alteraçōes histopatológicas em espécimes de MRC e de MEB*

Alterações histopatológicas

Cistos/ectasia ductal

Fibrose

Metaplasia apócrina

Hiperplasias epiteliais

Microcalcificações

Adenose esclerosante

Sem alterações

\section{Tipo do espécime}

MRC

$$
\begin{gathered}
43 / 72(59,7 \%) \\
23 / 72(31,9 \%) \\
18 / 72(25 \%) \\
31 / 72(43,1 \%) \\
10 / 72(13,9 \%) \\
3 / 72(4,2 \%) \\
11 / 72(15,3 \%)
\end{gathered}
$$

\section{MEB}

\begin{tabular}{c}
$440 / 867(50,9 \%)$ \\
$407 / 867(47,1 \%)$ \\
$213 / 867(24,6 \%)$ \\
$88 / 867(10,1 \%)$ \\
$34 / 867(3,9 \%)$ \\
$23 / 867(2,7 \%)$ \\
$238 / 867(27,5 \%)$ \\
\hline
\end{tabular}

*Havia mais de uma alteração histopatológica na maioria dos espécimes dos dois grupos. 


\section{Tabela 3 e de MEB}

Incidência das hiperplasias epiteliais em espécimes de MRC

Tipo de hiperplasia

Tipo do espécime

\begin{tabular}{lcc} 
& \multicolumn{1}{c}{ MRC } & MEB \\
HU & $24(77,5 \%)$ & $85(96,3 \%)$ \\
HDA & $1(3,2 \%)$ & $0(0 \%)$ \\
HDA + HU & $1(3,2 \%)$ & $0(0 \%)$ \\
NL/HLA & $2(6,5 \%)$ & $2(2,2 \%)$ \\
NL/HLA + HU & $1(3,2 \%)$ & $1(1,1 \%)$ \\
NL/HLA + HDA & $2(6,5 \%)$ & $0(0 \%)$ \\
Total & $\mathbf{3 1 ( 1 0 0 \% )}$ & $\mathbf{8 8 ( 1 0 0 \% )}$ \\
\hline
\end{tabular}

$H U$ = hiperplasia usual sem atipias; HDA = hiperplasia ductal atípica; $\mathrm{NL} / H L A=$ neoplasia lobular/hiperplasia lobular atípica. o desenvolvimento subseqüente de câncer de mama. Em meados da década de 1980, foi amplamente aceito que a maioria das mulheres com lesões benignas não-proliferativas da mama não possuía risco subseqüente aumentado para desenvolvimento de câncer de mama. No entanto, a presença de lesões hiperplásicas da mama está relacionada a aumento do risco relativo para desenvolver câncer de mama ${ }^{(8)}$. Esta correlação foi demonstrada a partir do estudo pioneiro de Dupont e Page (1985), que observaram que as portadoras de hiperplasias usuais sem atipias têm maior risco relativo para desenvolver câncer invasor subseqüente $(1,2$ a duas vezes), enquanto que as portadoras de hiperplasias atípicas apresentam risco aumentado em até quatro a cinco vezes ${ }^{(8)}$. Outros fatores como idade, tempo desde a biópsia, status menopausal e história familiar de câncer de mama também modificam o risco associado às lesões proliferativas $^{(24,26,32)}$.

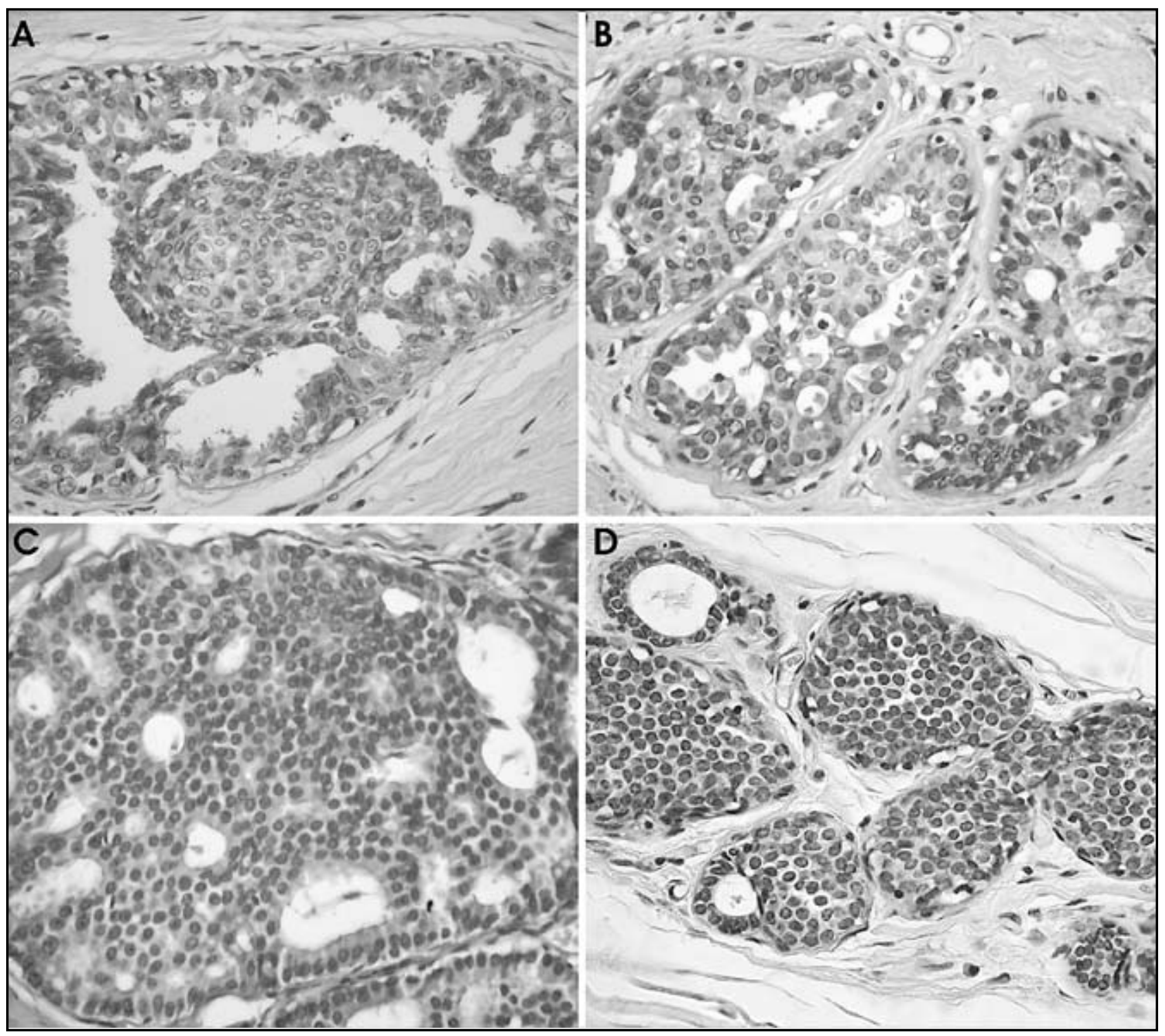

Figura - A e B: Hiperplasia usual sem atipias mostrando população celular heterogênea e fendas irregulares; C: hiperplasia ductal atípica apresentando células uniformes, espaços regulares. As células periféricas ainda apresentam polaridade preservada; $\mathbf{D}$ : hiperplasia lobular atípica/neoplasia lobular mostrando expansão parcial das unidades lobulares que são parcialmente preenchidas por células uniformes (hematoxilina e eosina: A, B e C, 200x, e D, 100x) 
O aumento da idade (acima de 45 anos) é considerado um fator de risco para o desenvolvimento de câncer de mama $^{(10)}$. Em nosso estudo, a maioria dos casos de hiperplasia epitelial foi de pacientes acima de 40 anos. A faixa etária de maior incidência de lesões proliferativas é após a quarta década de vida e próximo à menopausa $a^{(9,17,35)}$, e cerca de dez anos mais cedo que a faixa etária de maior incidência do câncer de mama ${ }^{(3)}$.

Apesar da dificuldade em se precisar a incidência das alterações fibrocísticas da mama na população em geral, estima-se que mais de $30 \%$ das mulheres entre 20 e 45 anos tenham alguma evidência clínica destas lesões no exame físico de rotina ${ }^{(1)}$. Em nosso estudo, $73,1 \%$ dos espécimes examinados eram de MEB e provenientes de mulheres clinicamente normais, tendo sido encontradas alterações histopatológicas em pelo menos uma de suas mamas. Estes achados estão de acordo com dados da literatura, onde alterações fibrocísticas em espécimes de MEB são detectadas em mais de $34 \%$ dos casos examinados ${ }^{(1,}$ $12,28,29,32,34)$. Em nosso estudo, grande parte das pacientes apresentou mais de um tipo de alteração histopatológica no mesmo espécime de mamoplastia. As principais alterações foram as do estroma, associadas ou não às do parênquima glandular. A freqüência das alterações fibrocísticas foi semelhante nos espécimes de MRC e MEB. A alta freqüência de alterações histológicas como cistos e fibrose estromal na maioria das mulheres, em grande parte assintomáticas, levou à mudança do conceito de doença fibrocística para alteração fibrocística. Estas alterações são hoje consideradas involutivas ou decorrentes de resposta exagerada à estimulação hormonal cíclica ${ }^{(16)}$.

Em nosso material, a incidência das alterações histopatológicas foi semelhante nos espécimes de MEB e MRC. Porém a incidência de lesões hiperplásicas foi maior nos casos de MRC (MEB $=10,1 \%$ e MRC $=43,1 \%)$, mesmo quando estas pacientes foram pareadas pela faixa etária. Estudos clínicos e epidemiológicos indicam que a maioria das mulheres com hiperplasias atípicas possui risco aumentado para desenvolvimento de câncer de mama ${ }^{(8,23,35)}$. A associação de lesões proliferativas da mama a outras lesões benignas pode aumentar o risco para o câncer de mama. Por exemplo, a presença de lesões proliferativas sem atipias e hiperplasia atípica em cicatriz radial pode aumentar o risco para o câncer de mama, principalmente em pacientes com mais de 50 anos $^{(18,31)}$.

O nosso interesse em estudar as mamoplastias contralaterais ao câncer deve-se ao fato de que mulheres com câncer de mama primário têm risco aumentado para desenvolver um segundo câncer na mama contralateral ${ }^{(15)}$. Em geral, este risco é de duas a seis vezes maior do que nas mulheres sem história prévia de câncer de mama ${ }^{(5)}$. No entanto, são raros os relatos na literatura que abordam a incidência de lesões proliferativas da mama em espécimes de $\mathrm{MRC}^{(30)}$.

Em nosso material, a incidência das hiperplasias epiteliais foi maior nas mulheres com idade superior a 40 anos, semelhante ao descrito na literatura ${ }^{(8,32)}$. A incidência de lesões pré-malignas ocorre cerca de dez a 15 anos antes da faixa etária de maior incidência do câncer de mama, cujo pico está entre 50 e 60 anos. Assim, a idade tem sido considerada um fator de risco para o desenvolvimento do câncer de mama. Esse risco pode ser modificado por uma série de fatores, como presença e tipo de hiperplasia epitelial, além da presença de neoplasia prévia da mama, como observado nas mulheres submetidas a MRC.

Em nossa série nenhum caso de carcinoma oculto foi detectado nos espécimes de MRC, embora estas mulheres tenham maior risco para desenvolver câncer de mama contralateral. No entanto, estudos de mastectomia profilática contralateral em mulheres pertencentes a grupos de alto risco, como pacientes com história familiar, mostram incidência de câncer de mama contralateral superior à da população em geral ${ }^{(20,37)}$. Nossos casos foram obtidos seqüencialmente da rotina de serviço de mastologia, a cirurgia contralateral teve indicação estética e não-preventiva e nossas pacientes não pertenciam a grupos de alto risco.

Além dos estudos morfológicos, trabalhos utilizando marcadores moleculares em lesões proliferativas pré-malignas têm sido feitos para tentar identificar mulheres com maior risco para desenvolvimento de carcinoma invasor. Estes estudos buscam marcadores que possam auxiliar o desenvolvimento de estratégias de prevenção mais eficientes do que aquelas baseadas somente em critérios clínicos, radiológicos e morfológicos ${ }^{(11,13,21)}$. Lesões precursoras pré-malignas possuem um perfil de expressão destes marcadores diferente do encontrado na mama normal, porém semelhante ao visto nos tumores invasivos ${ }^{(11,21)}$. Além disso, a detecção de marcadores moleculares de risco em lesões pré-malignas poderá ajudar, num futuro próximo, a definir um grupo de mulheres em que medidas preventivas seriam mais bem avaliadas quanto a seu custo/benefício.

No entanto, até o momento, as avaliações de risco ainda levam em conta apenas aspectos epidemiológicos e morfológicos. Estudos como o nosso, com avaliação criteriosa das lesões proliferativas aliada a outros fatores clínicos de risco, podem auxiliar a individualizar as pacientes e a definir estratégias de acompanhamento clínico ou adoção de medidas preventivas, como o uso de antiestrogênicos ou até medidas mais radicais, como a mastectomia profilática.

\section{Referências}

I. AYHAN, S. et al. Histologic profiles of the breast reduction specimens. Aesth Plast Surg, v. 26, p. 203-5, 2002.

2. BERNSTEIN, J. L. et al. Risk factors predicting the incidence of second primary breast cancer among women diagnosed with a first primary cancer. Am. J. Epidemiol, v. I36, p. $925-$ 36, 1992. 
3. BIGLIA, N. et al. Management of risk of breast carcinoma in postmenopausal women. Endocr Relat Câncer, v. I I, n. I, p. 69-83, 2004.

4. BROWN, M. H. et al. A cohort study of breast cancer risk in breast reduction patients. Plast Reconstr Surg, v. 103, n. 6, p. | 674-8I, 1999.

5. CLAUS, E. B. et al. The risk of a contralateral breast cancer among women diagnosed with ductal and lobular breast carcinoma in situ: data from the Connecticut Tumor Registry. Breast, v. I2, n. 6, p. 45I-6, 2003.

6. DE LA ROCHEFORDIÈRE,A. et al. Methachronous contralateral breast cancer as first event of relapse. Int J Radiation Oncol Phys, v. 36, n. 3, p. 615-21, 1996.

7. DE MASCAREL, l. et al. The incidence of cancer in contralateral reduction mammaplasty after mastectomy and reconstruction of removed breast. Tumori, v. 72, n. 2, p. 183-6, 1986.

8. DUPONT, W. D.; PAGE, D. L. Risk factors for breast cancer in women with proliferative breast disease. N Engl J Med, v. 312, p. |46-5।, 1985.

9. DUPONT,W. L.; PAGE, D. L. Breast cancer risk associated with proliferative disease, age at first birth, and a family history of breast cancer. Am J Epidemiol, v. I25, n. 5, p. 769-79, 1987.

I0. GAO, M. D. X. et al. Risk of second primary cancer in the contralateral breast in women treated for early-stage breast cancer: a population-based study. Int J Radiation Oncol Bio Phys, v. 56, n. 4, p. 1038-45, 2003.

I I. GOBBI, H. et al. Transforming growth factor-beta and breast cancer risk in women with mammary epithelial hyperplasia. J Natl Cancer Inst, v. 91, n. 24, p. 2096-101, 1999.

I2. GOBBI, H. et al. Estudo histopatológico de 1.000 peças cirúrgicas de mamoplastia redutora. Revista Médica de Minas Gerais, v. 2, n. 3, p. 146-8, 1992.

13. GOBBI, H. et al. Loss of expression of transforming growth factor beta type II receptor correlates with high tumour grade in human breast in-situ and invasive carcinomas. Histopathology, v. 36, n. 2, p. 168-77, 2000.

14. GOBBI, H. et al. Modelo informatizável para estudo anatomopatológico de peças cirúrgicas de mama. J Anatomopatologista, v. 8, n. 2, p. 14-6, 2001.

I 5. HISLOP,T.G. Second primary cancers of the breast: incidence and risk factors. Br J Cancer, v. 49, n. I, p. 79-85, 1984.

16. HUTTER, R. V. Goodbye to "fibrocystic disease". N Engl J Med, v. 312, n. 3, p. 179-8।, 1985.

17. ISHAG, M.T.et al. Pathologic findings in reduction mammaplasty specimens. Am J Clin Pathol, v. I20, n. 3, p. 377-80, 2003.

18. JACOBS, T. W. et al. Radial scars in benign breast-biopsy specimens and the risk of breast cancer. N Eng J Med, v. 340, n. 6, p. 430-6, 1999.

19. JANSCHEK, E. et al. Contralateral breast cancer: molecular differentiation between metastasis and second primary cancer. Breast Ca Re and Treat, v. 67, p. I-8, 200 I.
20. KHURANA, K. K. et al. Prophylactic mastectomy: pathologic findings in high-risk patients. Arch Pathol Lab Med, v. I24, p. 378-81, 2000.

21. KRISHNAMURTHY, S.; SNEIGE, N. Molecular and biologic markers of premalignant lesions of human breast. Advances in Anatomic Pathol, v. 9, n. 3, p. 185-97, 2002.

22. LANFREY, E. et al. Mammaplasty for symmetry of the contralateral breast and its oncologic value. Ann Chir Esthet, v. 42, n. 2, p. 160-7, 1997.

23. MARSHALL, L. M. et al. Risk of breast cancer associated with atypical hyperplasia of lobular and ductal types. Cancer Epidemiol Biomarkers Prev, v. 6, n. 5, p. 297-30I, 1997.

24. PAGE, D. L. et al. Premalignat and malignant disease of the breast: the roles of the pathologist. Mod Pathol, v. I I, n. 2, p. 120-8, 1998.

25. PAGE, D. L.; ANDERSON, T. J. Diagnostic histopathology of breast. Edinburgh: Churchill Livingstone, 1987.

26. PAGE, D. L.; DUPONT,W.D. Indicators of increased breast cancer risk in humans. J Cell Biochem Suppl, v. I6G, p. 175-82, 1992.

27.PETIT,J.Y.et al. Contralateral mastoplasty for breast reconstruction: a good opportunity for glandular exploration and occult carcinomas diagnosis. Ann Surg Oncol, v. 4, n. 6, p. 5। I-5, 1997.

28. PITANGUY, I.; TORRES, E. T.; ZELICOVICH, R. et al. Patologia em mamoplastia. Revista Brasileira de Cirurgia, v. 76, n. I, p. 45-5I, 1986.

29. QUIRINO, I. L. G. et al. Alteração histopatológica em espécimes de mamoplastia redutora contralateral (MRC) a câncer de mama. Jornal Brasileiro de Patologia, v. 37, n. 2, p. 94, 2001.

30. ROUBIDOUX, M.A. et al. Women with breast cancer: histologic findings in the contralateral breast. Radiology, v. 203, n. 3, p. 69I-4, 1997.

3I. SANDERS, M. E. et al. Interdependence of radial scar and proliferative disease and with respect to invasive breast cancer risk in benign breast biopsies, Mod Pathol [Abstract], v. I5, p. 50, 2002.

32. SCHNITT, S. J. Benign breast disease and breast cancer risk. Am J Surg Pathol, v. 27, n. 6, p. 836-4I, 2003.

33. TABÁR, L. et al. Beyond randomized controlled trials. Cancer, v. 9I, p. 1724-31, 2001.

34. TANG, C. L. et al. Breast cancer found at the time of breast reduction. Plast Reconst Surgery, v. 103, n. 6, p. 1682-6, 1999.

35. TAVASSOLI, F. A.; NORRIS, H. J. A comparison of the results of long-term follow-up for atypical intraductal hyperplasia and intraductal hyperplasia of the breast. Cancer, v. 65, n. 3, p. 5 I 8-29, 1990.

36. TAVASSOLI, F. A.; DEVILEE, P. (eds.). World Health Organization Classification of Tumors. Pathology and Genetics of Tumors of the Breast and Female Genital Organs. IARC Press: Lyon, 2003.

37. VAN GEEL, A. N. Prophylactic mastectomy: the Rotterdam experience. The Breast, v. 12, p. 357-61, 2003. 